\title{
Two-loop leading color corrections to heavy-quark pair production in the gluon fusion channel
}

\section{R. Bonciani, ${ }^{a}$ A. Ferroglia, ${ }^{b}$ T. Gehrmann, ${ }^{c}$ A. von Manteuffel ${ }^{c}$ and C. Studerus ${ }^{d}$}

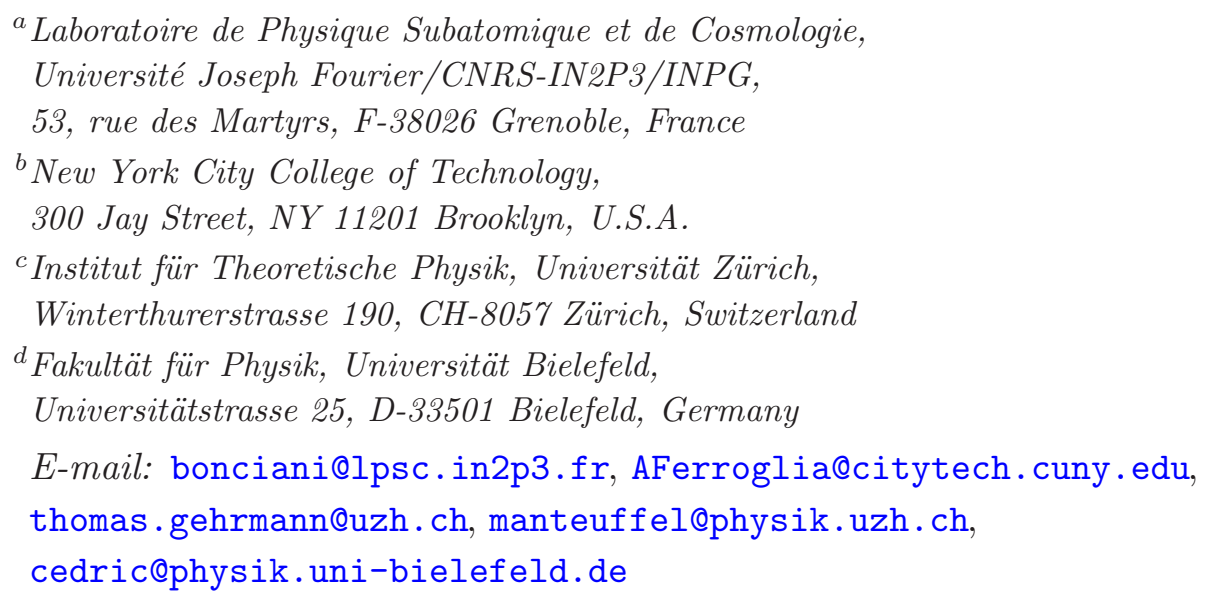

ABSTRACT: We evaluate the two-loop QCD diagrams contributing to the leading color coefficient of the heavy-quark pair production cross section in the gluon fusion channel. We obtain an analytic expression, which is valid for any value of the Mandelstam invariants $s$ and $t$ and of the heavy-quark mass $m$. Our findings agree with previous analytic results in the small-mass limit and with recent results for the coefficients of the IR poles.

Keywords: NLO Computations, Hadronic Colliders, QCD

ARXIV EPRINT: 1011.6661 


\section{Contents}

1 Introduction $\quad 1$

2 Notation and conventions 3

3 Calculation 4

4 Renormalization 5

$\begin{array}{lll}5 & \text { Results } & 7\end{array}$

6 Conclusions and outlook $\quad 10$

\section{Introduction}

The top quark is the heaviest elementary particle known to date. Its large mass of $173 \mathrm{GeV}$ makes it a crucial tool for the study of the electroweak symmetry breaking mechanism and for the search of signals of physics beyond the Standard Model. The study of the properties of the top quark at the Tevatron during the last 15 years provided precise measurements [1$4]$ of the top-quark mass (with a relative error of $0.61 \%$ ) and of the total top-quark pair production cross section (with a relative error of about 10\%). Owing to the by-now large Tevatron dataset, measurements of differential distributions in top pair production are becoming possible [5-7]. A new set of precise experimental measurements is expected to be obtained by the LHC experiments soon. At the LHC, running at $7 \mathrm{TeV}$, with an integrated luminosity of only $\sim 200 \mathrm{pb}^{-1}$, it should be possible to record about 30000 top quark pair events before selection [8]. With the planned increases in center of mass energy and luminosity, the LHC will turn into a veritable top-quark factory, producing millions of top-quark events per year [9].

In order to take full advantage of the improved experimental measurements, it will be necessary to provide theoretical predictions for the measured observables which are as accurate as possible. Most of the observables related to the top-quark pair production have been calculated up to NLO [10-24]. In several cases, the next-to-leading (NLO) corrections have been supplemented by the resummation of large logarithmic corrections at leading (LL, [25-31]), next-to-leading (NLL, [32-38]) and next-to-next-to-leading logarithmic (NNLL, [39-43]) accuracy. However, to match the precision of the forthcoming experimental data, full next-to-next-to-leading order (NNLO) calculations are required for at least some of the observables, such as the top-quark pair production total cross section $[44-48]$. 
To obtain NNLO predictions for the top quark pair production in QCD, the following matrix elements need to be computed: i) two-loop matrix elements in both the quarkantiquark annihilation channel and the gluon-fusion channel; ii) one-loop matrix elements with one extra parton in the final state; and iii) tree-level matrix elements with two extra partons in the final state. The diagrams belonging to the sets ii) and iii) form part of the NLO corrections to top-pair-plus-jet production and have been calculated by several groups [49-52]. Aside from the calculation of the required Feynman diagrams, a full NNLO calculation of the top-quark pair production cross section is particularly challenging because it entails the development of a NNLO subtraction method, which could be used in processes with massive partons [53-69].

As far as matrix elements are concerned, the last missing ingredient to the NNLO corrections to top quark pair production are the two-loop matrix elements for $q \bar{q} \rightarrow t \bar{t}$ and $g g \rightarrow t \bar{t}$. Both types of matrix elements were calculated in the $s \gg m^{2}$ limit (where $s$ is the partonic center of mass energy and $m$ is the mass of the top quark) [70,71]. A fully numerical calculation of the two-loop corrections in the quark-antiquark annihilation channel is also available [72].

This paper is the third of a series of works aiming towards the analytic calculation of the two-loop matrix elements for the top pair production process. For what concerns the quark-antiquark annihilation channel, the two-loop diagrams involving a closed light or heavy-quark loop were evaluated in [73], while the two-loop diagrams contributing to the leading color coefficient were evaluated in [74]. In both cases, the results obtained retain the full dependence on the top-quark mass and on the kinematic invariants; they agree with the numerical results of [72]. Having analytical results available has several advantages over a purely numerical representation. Besides their considerably shorter evaluation time, the analytical results also allow for an expansion in different kinematical limits (threshold, high energy).

In the present paper, an analytical expression for the two-loop diagrams contributing to the leading color coefficient in the gluon-fusion channel is derived. We carry out the calculation by employing the technique based on the Laporta algorithm [75-78] and the differential equation method [79-86], already used in [73, 74]. The calculation of the leading color coefficient in the gluon fusion does not require the calculation of any new master integrals beyond the ones obtained in the two previous works, such that we do not discuss the calculational method in full detail. The interested reader can find in $[73,74]$ a detailed description of the techniques employed.

The paper is organized as follows: in section 2, we introduce our notation and conventions; in section 3, we summarize the most relevant features of our calculational method. Section 4 describes the UV renormalization of the bare amplitude. The resulting two-loop amplitude contributions are described in section 5, where we also provide numerical values in some benchmark points, and discuss the expansion in the threshold limit. Finally, section 6 contains our conclusions. 


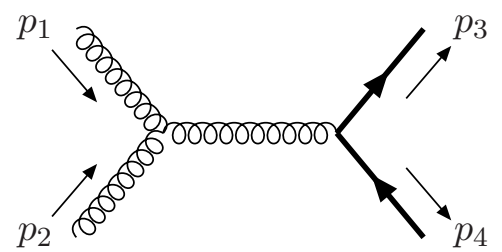

(a)

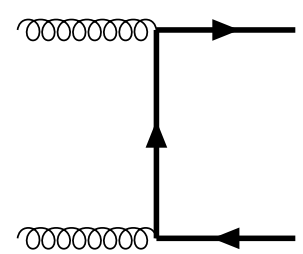

(b)

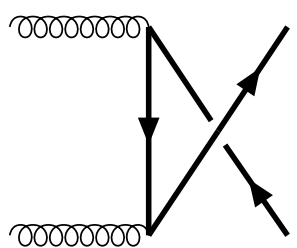

(c)

Figure 1. Tree-level amplitude. Massive quarks are indicated by a thick line.

\section{Notation and conventions}

We consider the scattering process

$$
g\left(p_{1}\right)+g\left(p_{2}\right) \longrightarrow t\left(p_{3}\right)+\bar{t}\left(p_{4}\right)
$$

in Euclidean kinematics, where $p_{i}^{2}=0$ for $i=1,2$ and $p_{j}^{2}=-m^{2}$ for $j=3,4$. The Mandelstam variables are defined as follows

$$
s=-\left(p_{1}+p_{2}\right)^{2}, \quad t=-\left(p_{1}-p_{3}\right)^{2}, \quad u=-\left(p_{1}-p_{4}\right)^{2} .
$$

Conservation of momentum implies that $s+t+u=2 m^{2}$.

The squared matrix element (summed over spin and color), calculated in $d=4-2 \varepsilon$ dimensions, can be expanded in powers of the strong coupling constant $\alpha_{S}$ as follows:

$$
\sum|\mathcal{M}|^{2}(s, t, m, \varepsilon)=16 \pi^{2} \alpha_{S}^{2}\left[\mathcal{A}_{0}+\left(\frac{\alpha_{s}}{\pi}\right) \mathcal{A}_{1}+\left(\frac{\alpha_{s}}{\pi}\right)^{2} \mathcal{A}_{2}+\mathcal{O}\left(\alpha_{s}^{3}\right)\right]
$$

The tree-level amplitude involves the three diagrams shown in figure 1 and their contribution to eq. (2.3) is given by

$$
\begin{aligned}
\mathcal{A}_{0}= & \left(N_{c}^{2}-1\right)\left\{\left[\frac{1}{N_{c}} \frac{1}{t_{1}^{2} u_{1}^{2}}-N_{c} \frac{\left(t_{1}^{2}+u_{1}^{2}\right)}{t_{1}^{2} u_{1}^{2}\left(t_{1}+u_{1}\right)^{2}}\right] F_{1}\left(t_{1}, u_{1}, m^{2}\right)+\varepsilon\left[\frac{1}{N_{c}} \frac{4\left(t_{1}^{2}+u_{1} t_{1}+u_{1}^{2}\right)}{t_{1} u_{1}}\right.\right. \\
& \left.\left.-N_{c} \frac{4\left(t_{1}^{2}+u_{1}^{2}\right)\left(t_{1}^{2}+u_{1} t_{1}+u_{1}^{2}\right)}{t_{1} u_{1}\left(t_{1}+u_{1}\right)^{2}}\right]-\varepsilon^{2}\left[\frac{1}{N_{c}} \frac{2\left(t_{1}+u_{1}\right)^{2}}{t_{1} u_{1}}-N_{c} \frac{2\left(t_{1}^{2}+u_{1}^{2}\right)}{t_{1} u_{1}}\right]\right\}
\end{aligned}
$$

where $N_{c}$ is the number of colors, $t_{1}=t-m^{2}, u_{1}=u-m^{2}$, and

$$
F_{1}\left(t_{1}, u_{1}, m\right)=-2 t_{1} u_{1}\left(t_{1}^{2}+u_{1}^{2}\right)+8 m^{2} t_{1} u_{1}\left(t_{1}+u_{1}\right)+8 m^{4}\left(t_{1}+u_{1}\right)^{2} .
$$

The $\mathcal{O}\left(\alpha_{S}\right)$ term $\mathcal{A}_{1}$ in eq. (2.3) arises from the interference of one-loop diagrams with the tree-level amplitude [10-24]. The $\mathcal{O}\left(\alpha_{S}^{2}\right)$ term $\mathcal{A}_{2}$ consists of two parts, the interference of two-loop diagrams with the Born amplitude and the interference of one-loop diagrams among themselves:

$$
\mathcal{A}_{2}=\mathcal{A}_{2}^{(2 \times 0)}+\mathcal{A}_{2}^{(1 \times 1)}
$$



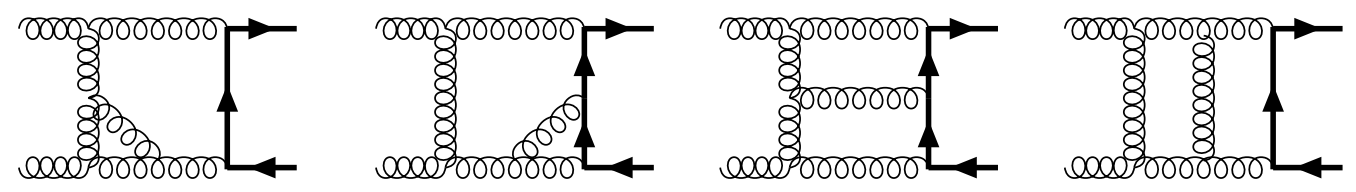

Figure 2. Some of the two-loop planar box diagrams involved in the calculation.

The term $\mathcal{A}_{2}^{(1 \times 1)}$ was derived in [87-90]. $\mathcal{A}_{2}^{(2 \times 0)}$, originating from the two-loop diagrams, can be decomposed according to color and flavor structures as follows:

$$
\begin{aligned}
\mathcal{A}_{2}^{(2 \times 0)}=\left(N_{c}^{2}-1\right) & \left\{N_{c}^{3} A+N_{c} B+\frac{1}{N_{c}} C+\frac{1}{N_{c}^{3}} D+N_{c}^{2} N_{l} E_{l}+N_{c}^{2} N_{h} E_{h}+N_{l} F_{l}+N_{h} F_{h}\right. \\
& +\frac{N_{l}}{N_{c}^{2}} G_{l}+\frac{N_{h}}{N_{c}^{2}} G_{h}+N_{c} N_{l}^{2} H_{l}+N_{c} N_{h}^{2} H_{h}+N_{c} N_{l} N_{h} H_{l h}+\frac{N_{l}^{2}}{N_{c}} I_{l} \\
& \left.+\frac{N_{h}^{2}}{N_{c}} I_{h}+\frac{N_{l} N_{h}}{N_{c}} I_{l h}\right\},
\end{aligned}
$$

where $N_{l}$ and $N_{h}$ are the number of light- and heavy-quark flavors, respectively. The coefficients $A, B, \ldots, I_{l h}$ in eq. (2.6) are functions of $s, t$, and $m$, as well as of the dimensional regulator $\varepsilon$. These quantities were calculated in [71] in the approximation $s,|t|,|u| \gg m^{2}$. For a fully differential description of top quark pair production at NNLO, the complete mass dependence of $\mathcal{A}_{2}^{(2 \times 0)}$ is required; to date no such a result is available. Recently, a formula which allows to calculate the IR poles of a generic two-loop QCD amplitude was derived [91, 92] and employed to obtain analytic expressions for all of the IR poles for the top-quark pair production [93].

In this work, we provide an exact analytic expression for the leading colour coefficient $A$ in eq. (2.6), which receives contributions from planar Feynman diagrams only.

\section{Calculation}

The package QGRAF [94] generates 789 two-loop Feynman diagrams contributing to the process $g g \rightarrow t \bar{t}$ (considering one massless and one massive flavor). Evaluating their color structures, we find that 300 of them contribute to the leading color coefficient in eq. (2.6) (some of the box diagrams involved in the calculation are shown in figure 2). Since we use the covariant sum over the polarizations of the incoming gluons, we have to consider additional 116 (out of 218) diagrams for the process initiated by ghosts. We interfere the two-loop diagrams with the tree-level amplitude, and calculate the color and Dirac traces before carrying out the integration over the loop momenta. The resulting scalar loop integrals are reduced to a set of Master Integrals (MIs) employing the technique based upon the Laporta algorithm [75-78]. Then, the MIs can be evaluated analytically by means of the differential equation method [79-86].

Starting with the QGRAF output, the calculation of the interferences in terms of the MIs was carried out with a parallelized C++ package: Reduze 2 [95]. In particular, this 
code provides a fully distributed variant of the Laporta algorithm for the reduction ${ }^{1}$ of the loop integrals. The package employs GiNaC [99] for the algebraic manipulations.

The MIs needed for the calculation presented in this paper were already known in the literature [73, 74, 100-113]. In particular, all of the two-loop four-point MIs encountered in the calculation of the leading color coefficient in the gluon-fusion channel coincide with the ones needed for the corresponding calculation in the quark-antiquark annihilation channel [74], or can be obtained by the latter by replacing the Mandelstam variable $t$ with $u$. All of the MIs were calculated in the non-physical region $s<0$, where they are real. The result in the physical region is then recovered by analytic continuation.

The transcendental functions appearing in the results are one- and two-dimensional harmonic polylogarithms (HPLs) [107, 108, 114-123] of maximum weight four. All of the HPLs appearing in the analytic expression of the coefficient $A$ can be evaluated numerically with arbitrary precision by employing the methods and codes described in [120]. A detailed list of the functional basis employed in this calculation can be found in appendix A of [74]. Appendix B in the same paper describes the expansion of this class of HPLs in the threshold limit $\beta \rightarrow 0$, which requires some care. As expected, the sum of the bare two-loop corrections (as well as the UV renormalized corrections) is symmetric with respect to the exchange $t \leftrightarrow u$.

\section{Renormalization}

The renormalized QCD amplitude is obtained from the bare one by expanding the following expression:

$$
\begin{aligned}
\mathcal{A}_{\mathrm{ren}} & =\prod_{n} Z_{\mathrm{WF}, n}^{1 / 2} \mathcal{A}_{\text {bare }}\left(\alpha_{S, \text { bare }} \rightarrow Z_{\alpha_{S}} \alpha_{S}, m_{\text {bare }} \rightarrow Z_{m} m\right), \\
& =Z_{\mathrm{WF}, g} Z_{\mathrm{WF}, t} \mathcal{A}_{\text {bare }}\left(\alpha_{S, \text { bare }} \rightarrow Z_{\alpha_{S}} \alpha_{S}, m_{\text {bare }} \rightarrow Z_{m} m\right),
\end{aligned}
$$

where $Z_{\mathrm{WF}, n}(n=g, t)$ are the external particle wave-function renormalization factors, $\alpha_{S}$ is the renormalized coupling constant, $Z_{\alpha_{S}}$ is the coupling constant renormalization factor, $m$ is the renormalized heavy-quark mass, and $Z_{m}$ is the mass renormalization factor. In the rest of the section we suppress the subscript " $S$ " in $\alpha_{S}$.

We introduce the following auxiliary quantities:

$$
a_{0}=\frac{\alpha_{\text {bare }}}{\pi}, \quad \text { and } \quad a=\frac{\alpha}{\pi} .
$$

By expanding the amplitude and the wave function renormalization factor in $a_{0}$ we find:

$$
\begin{aligned}
\mathcal{A}_{\text {ren }}\left(\alpha_{\text {bare }}\right) & =a_{0} \mathcal{A}_{0}+a_{0}^{2} \mathcal{A}_{1}+a_{0}^{3} \mathcal{A}_{2}+\mathcal{O}\left(a_{0}^{4}\right), \\
Z_{\mathrm{WF}, n} & =1+a_{0} \delta Z_{\mathrm{WF}, n}^{(1)}+a_{0}^{2} \delta Z_{\mathrm{WF}, n}^{(2)}+\mathcal{O}\left(a_{0}^{3}\right), \quad(n=g, t), \\
Z_{m} & =1+a_{0} \delta Z_{m}^{(1)}+a_{0}^{2} \delta Z_{m}^{(2)}+\mathcal{O}\left(a_{0}^{3}\right) .
\end{aligned}
$$

\footnotetext{
${ }^{1}$ Other reduction codes released publicly are the Maple package A.I.R. [96], the Mathematica package FIRE [97] and the C++ package Reduze [98].
} 
The relation between $a_{0}$ and $a$ is given by:

$$
a_{0}=a+a^{2} \delta Z_{\alpha}^{(1)}+a^{3} \delta Z_{\alpha}^{(2)}+\mathcal{O}\left(a^{4}\right)
$$

By employing eqs. (4.4), (4.5) in eq. (4.2) we find

$$
\begin{aligned}
\mathcal{A}_{\mathrm{ren}}= & a \mathcal{A}_{0}+a^{2} \mathcal{A}_{\mathrm{ren}}^{(1)}+a^{3} \mathcal{A}_{\mathrm{ren}}^{(2)}+\mathcal{O}\left(a^{4}\right) \\
\mathcal{A}_{\mathrm{ren}}^{(1)}= & \mathcal{A}_{1}+\left(\delta Z_{\mathrm{WF}, t}^{(1)}+\delta Z_{\mathrm{WF}, g}^{(1)}+\delta Z_{\alpha}^{(1)}\right) \mathcal{A}_{0}-\delta Z_{m}^{(1)} \mathcal{A}_{0}^{(\text {mass } \mathrm{cr})} \\
\mathcal{A}_{\mathrm{ren}}^{(2)}= & \mathcal{A}_{2}+\left(\delta Z_{\mathrm{WF}, t}^{(1)}+\delta Z_{\mathrm{WF}, g}^{(1)}+2 \delta Z_{\alpha}^{(1)}\right) \mathcal{A}_{1}+\left(\delta Z_{\mathrm{WF}, t}^{(1)} \delta Z_{\mathrm{WF}, g}^{(1)}+2 \delta Z_{\mathrm{WF}, t}^{(1)} \delta Z_{\alpha}^{(1)}\right. \\
& \left.+2 \delta Z_{\mathrm{WF}, g}^{(1)} \delta Z_{\alpha}^{(1)}+\delta Z_{\mathrm{WF}, t}^{(2)}+\delta Z_{\mathrm{WF}, g}^{(2)}+\delta Z_{\alpha}^{(2)}\right) \mathcal{A}_{0}-\delta Z_{m}^{(1)} \mathcal{A}_{1}^{(\text {mass } \mathrm{CT})} \\
& -\left(\delta Z_{m}^{(2)}+2 \delta Z_{\alpha}^{(1)} \delta Z_{m}^{(1)}+\delta Z_{\mathrm{WF}, t}^{(1)} \delta Z_{m}^{(1)}\right) \mathcal{A}_{0}^{(\text {mass } \mathrm{CT})}+\left(\delta Z_{m}^{(1)}\right)^{2} \mathcal{A}_{0}^{(2 \text { mass } \mathrm{CT})} .
\end{aligned}
$$

In the equations above, $\mathcal{A}_{i}, i=0,1,2$, represents the bare $i$-loop amplitude stripped of the factor $a, \mathcal{A}_{0}^{(\text {mass Cr) }}$ represents the amplitude obtained by adding a mass insertion to the virtual top propagator in diagrams (b) and (c) of figure $1 . \mathcal{A}_{1}^{\text {(mass ст) }}$ can be obtained by considering all possible mass insertions in the one-loop diagrams. Finally, $\mathcal{A}_{0}^{(2 \text { mass ст) }}$ is obtained by adding two mass insertions to the virtual propagator in diagrams (b) and (c) of figure 1.

In this work we employ a mixed renormalization scheme in which the wave functions and the heavy-quark mass are renormalized on shell, while the strong coupling constant is renormalized in the $\overline{\mathrm{MS}}$ scheme. The explicit expressions of the one-loop renormalization factors in eq. (4.6) are:

$$
\begin{aligned}
\delta Z_{\mathrm{WF}, t}^{(1)} & =C(\varepsilon)\left(\frac{\mu^{2}}{m^{2}}\right)^{\varepsilon} C_{F}\left(-\frac{3}{4 \varepsilon}-\frac{1}{1-2 \varepsilon}\right) \\
\delta Z_{\mathrm{WF}, g}^{(1)} & =0 \\
\delta Z_{\alpha}^{(1)} & =C(\varepsilon) \frac{e^{-\gamma \varepsilon}}{\Gamma(1+\varepsilon)}\left(-\frac{\beta_{0}}{2 \varepsilon}\right) \\
\delta Z_{m}^{(1)} & =\delta Z_{\mathrm{WF}, t}^{(1)}
\end{aligned}
$$

where $C(\varepsilon)=(4 \pi)^{\varepsilon} \Gamma(1+\varepsilon), \beta_{0}=(11 / 6) C_{A}-(1 / 3)\left(N_{l}+N_{h}\right)$ and where $\gamma$ is the EulerMascheroni constant $\gamma \approx 0.577216$.

Concerning the two-loop renormalization factors, we provide only the terms which contribute to the renormalization of the leading color coefficient. They are (see for in- 
stance [124]):

$$
\begin{aligned}
\delta Z_{\mathrm{WF}, t}^{(2)}= & C(\varepsilon)^{2}\left(\frac{\mu^{2}}{m^{2}}\right)^{2 \varepsilon} C_{F}\left[C_{F}\left(\frac{9}{32 \varepsilon^{2}}+\frac{51}{64 \varepsilon}+\frac{433}{128}-\frac{3}{2} \zeta(3)+6 \zeta(2) \ln 2-\frac{39}{8} \zeta(2)\right)\right. \\
& \left.+C_{A}\left(-\frac{11}{32 \varepsilon^{2}}-\frac{101}{64 \varepsilon}-\frac{803}{128}+\frac{3}{4} \zeta(3)-3 \zeta(2) \ln 2+\frac{15}{8} \zeta(2)\right)+\mathcal{O}(\varepsilon)\right], \\
\delta Z_{\mathrm{WF}, g}^{(2)}= & 0, \\
\delta Z_{\alpha}^{(2)}= & C(\varepsilon)^{2}\left(\frac{e^{-\gamma \varepsilon}}{\Gamma(1+\varepsilon)}\right)^{2} \frac{1}{4 \varepsilon}\left[\left(\frac{11}{6}\right)^{2} \frac{C_{A}^{2}}{\varepsilon}-\frac{17}{12} C_{A}^{2}\right] \\
\delta Z_{m}^{(2)}= & C(\varepsilon)^{2}\left(\frac{\mu^{2}}{m^{2}}\right)^{2 \varepsilon} C_{F}\left[C_{F}\left(\frac{9}{32 \varepsilon^{2}}+\frac{45}{64 \varepsilon}+\frac{199}{128}-\frac{15}{8} \zeta(2)-\frac{3}{4} \zeta(3)+3 \zeta(2) \ln 2\right)\right. \\
& \left.+C_{A}\left(-\frac{11}{32 \varepsilon^{2}}-\frac{91}{64 \varepsilon}-\frac{605}{128}+\frac{1}{2} \zeta(2)+\frac{3}{8} \zeta(3)-\frac{3}{2} \zeta(2) \ln 2\right)+\mathcal{O}(\varepsilon)\right]
\end{aligned}
$$

\section{Results}

The main result of the present paper is an analytic expression for the coefficient $A$ in eq. (2.6) which retains the complete dependence on the variables $t, u$, on the renormalization scale $\mu$, and on the top-quark mass $m$. The analytic result is too long to be explicitly presented in written form. To make it available to the reader we include in the arXiv submission of this work a text file with the complete result. The coefficients in eq. (2.6) still contain infrared poles. This makes our result dependent on the choice of a global, $\varepsilon$-dependent normalization factor. We choose to present the coefficient $A$ factoring out an overall term

$$
C^{2}(\varepsilon)=\left[(4 \pi)^{\varepsilon} \Gamma(1+\varepsilon)\right]^{2} .
$$

Another possible normalization, widely used in the literature, is the one in which a term

$$
\tilde{C}^{2}(\varepsilon)=\left[(4 \pi)^{\varepsilon} e^{-\gamma \varepsilon}\right]^{2}
$$

is retained as an overall factor (see for instance [93] and [72]). Our result, therefore, can be compared to the results in $[72,93]$ by multiplying it by a coefficient

$$
e^{2 \gamma \varepsilon} \Gamma^{2}(1+\varepsilon)=1+\zeta(2) \varepsilon^{2}-\frac{2}{3} \zeta(3) \varepsilon^{3}+\frac{7}{10} \zeta(2)^{2} \varepsilon^{4}+\mathcal{O}\left(\varepsilon^{5}\right)
$$

While the formulae in the text and the supplied files are given in the normalization defined by eq. (5.1), for ease of comparison with other results in the literature we present in table 1 and in figures 3-4 a few benchmark points and plots in the normalization of eq. (5.2).

We provide a code which numerically evaluates the analytic expression of the leading color coefficient for arbitrary values of the mass scales involved in the calculation. The code is written in $\mathrm{C}++$ and uses the package for the evaluation of multiple polylogarithms within GiNaC [120]. The typical time for the evaluation of the leading color coefficient in a point of the phase space in double precision accuracy is $0.5 \mathrm{~s}$ on a $2.8 \mathrm{GHz}$ core. 


\begin{tabular}{|c|c|c|c|c|c|c|c|}
\hline$\frac{s}{m^{2}}$ & $\frac{t}{m^{2}}$ & $\frac{\mu}{m}$ & $1 / \varepsilon^{4}$ & $1 / \varepsilon^{3}$ & $1 / \varepsilon^{2}$ & $1 / \varepsilon$ & $\varepsilon^{0}$ \\
\hline 5 & -1.25 & 1 & 10.749426 & 18.693893 & -156.82372 & 262.14826 & 12.721807 \\
\hline 43 & -21 & 1.7 & 9.3642942 & -27.358589 & -41.372387 & 305.80422 & -707.01281 \\
\hline 8.1 & -0.6 & 2.1 & 27.306074 & 138.38466 & -381.11526 & -571.56293 & 1859.6594 \\
\hline
\end{tabular}

Table 1. Numerical values of the coefficients appearing in the $\varepsilon$ expansion of $A$ in the normalization that factors out the term $\left[(4 \pi)^{\varepsilon} e^{-\gamma \varepsilon}\right]^{2}$.

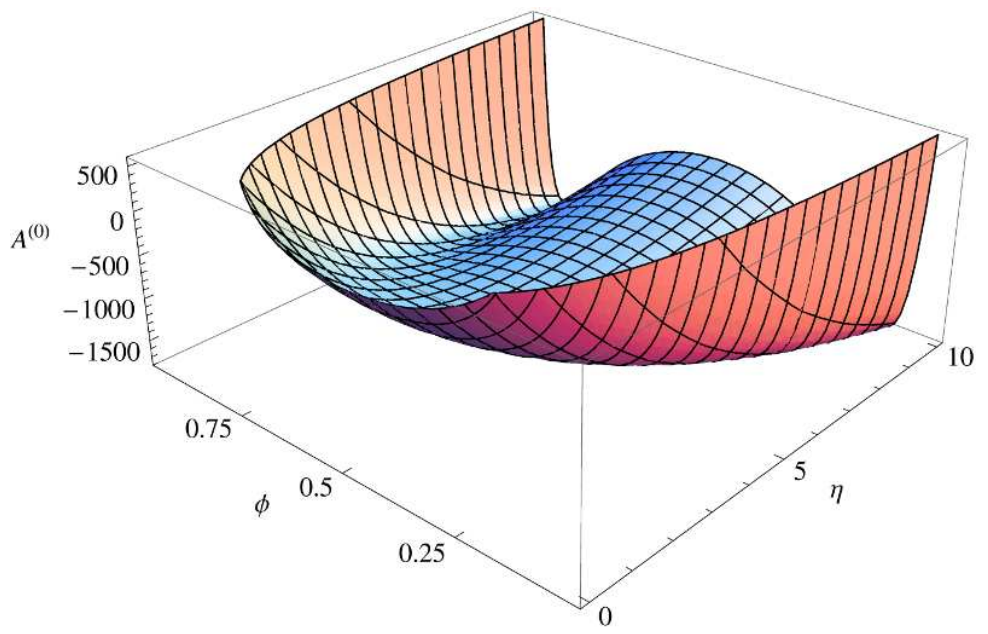

Figure 3. Finite part of the coefficient $A$ as a function of the variables $\eta \equiv s /\left(4 m^{2}\right)-1$ and $\phi \equiv-\left(t-m^{2}\right) / s$. We employed the normalization that factors out the term $\left[(4 \pi)^{\varepsilon} e^{-\gamma \varepsilon}\right]^{2}$ and set $\mu=m$.

Our result was cross checked by comparing it with the partial results already available in the literature. By expanding the analytic expression of the coefficient $A$ in the limit $s,|t|,|u| \gg m^{2}$ and neglecting terms suppressed by positive powers of $m^{2} / s$ for $t / s=$ const, we obtained the result published in [71]. For the IR poles of the coefficient $A$ we find analytic agreement with the results of [93].

In figure 3 the finite part of $A$ is plotted as a surface depending on the variables $\eta$ and $\phi$, defined as

$$
\eta=\frac{s}{4 m^{2}}-1, \quad \phi=-\frac{t-m^{2}}{s}, \quad \frac{1}{2}\left(1-\sqrt{\frac{\eta}{1+\eta}}\right) \leq \phi \leq \frac{1}{2}\left(1+\sqrt{\frac{\eta}{1+\eta}}\right) .
$$

Finally, it is possible to expand the expression for $A$ for values of the center of mass energy close to the production threshold. We define

$$
\beta=\sqrt{1-\frac{4 m^{2}}{s}}, \quad \xi=\frac{1-\cos \theta}{2}, \quad L_{\mu}=\ln \left(\frac{\mu^{2}}{m^{2}}\right),
$$

where $\theta$ is the scattering angle in the partonic center of mass frame. Keeping $\xi=$ const, we expand our results in powers of the heavy quark velocity $\beta$ up to terms of order $\beta^{5}$ included. The coefficients of this expansion contain transcendental constants which originate from 
one- and two-dimensional HPLs evaluated at $x=1$. Since we did not find a satisfactory analytical representation for all of these constants, in the formulae below we provide the coefficients of the expansion in a numerical form. We find:

$$
A(\beta, \xi)=\frac{A^{(-4)}(\beta, \xi)}{\varepsilon^{4}}+\frac{A^{(-3)}(\beta, \xi)}{\varepsilon^{3}}+\frac{A^{(-2)}(\beta, \xi)}{\varepsilon^{2}}+\frac{A^{(-1)}(\beta, \xi)}{\varepsilon}+A^{(0)}(\beta, \xi)+\mathcal{O}(\varepsilon)
$$

with

$$
\begin{aligned}
A^{(-4)}= & +[24-32 \xi(1-\xi)] \beta^{2}+\left[32-128 \xi(1-\xi)-256(\xi(1-\xi))^{2}\right] \beta^{4} \\
& +\mathcal{O}\left(\beta^{6}\right) \\
A^{(-3)}= & 13.1526+16 L_{\mu}+\left[71.4579+48 L_{\mu}-\left(84.6105+64 L_{\mu}\right) \xi(1-\xi)\right] \beta^{2} \\
& +\left[103.277+64 L_{\mu}-\left(487.775+256 L_{\mu}\right) \xi(1-\xi)\right. \\
& \left.-\left(1850.22+512 L_{\mu}\right)(\xi(1-\xi))^{2}\right] \beta^{4}+\mathcal{O}\left(\beta^{6}\right), \\
A^{(-2)}= & -155.954-32.3614 L_{\mu}+16 L_{\mu}^{2} \\
& -\left[388.711+33.0843 L_{\mu}-48 L_{\mu}^{2}-\left(1148.69+65.4457 L_{\mu}-64 L_{\mu}^{2}\right) \xi(1-\xi)\right] \beta^{2} \\
& -\left[461.697+28.1123 L_{\mu}-64 L_{\mu}^{2}-\left(2976.85-36.884 L_{\mu}-256 L_{\mu}^{2}\right) \xi(1-\xi)\right. \\
& \left.+\left(2814.61+1823.1 L_{\mu}+512 L_{\mu}^{2}\right)(\xi(1-\xi))^{2}\right] \beta^{4}+\mathcal{O}\left(\beta^{6}\right), \\
A^{(-1)}= & 224.144-191.469 L_{\mu}-61.6948 L_{\mu}^{2}+10.6667 L_{\mu}^{3} \\
& +\left[117.543-592.101 L_{\mu}-121.084 L_{\mu}^{2}+32 L_{\mu}^{3}\right. \\
& \left.-\left(774.295-1463.61 L_{\mu}-182.779 L_{\mu}^{2}+42.6667 L_{\mu}^{3}\right) \xi(1-\xi)\right] \beta^{2} \\
& -\left[92.4819+744.745 L_{\mu}+145.446 L_{\mu}^{2}-42.6667 L_{\mu}^{3}\right. \\
& +\left(553.64-4574.2 L_{\mu}-432.449 L_{\mu}^{2}+170.667 L_{\mu}^{3}\right) \xi(1-\xi) \\
& \left.-\left(17463.5+2328.24 L_{\mu}-884.435 L_{\mu}^{2}-341.333 L_{\mu}^{3}\right)(\xi(1-\xi))^{2}\right] \beta^{4} \\
& +\mathcal{O}\left(\beta^{6}\right), \\
A^{(0)}= & 403.869+787.434 L_{\mu}-77.4706 L_{\mu}^{2}-50.9076 L_{\mu}^{3}+5.33333 L_{\mu}^{4} \\
& +\left[1627.76+1320.97 L_{\mu}-338.107 L_{\mu}^{2}-110.056 L_{\mu}^{3}+16 L_{\mu}^{4}\right. \\
& \left.-\left(8363.19+4515.06 L_{\mu}-831.618 L_{\mu}^{2}-160.964 L_{\mu}^{3}+21.3333 L_{\mu}^{4}\right) \xi(1-\xi)\right] \beta^{2} \\
& +\left[1826.76+1164.29 L_{\mu}-440.308 L_{\mu}^{2}-136.075 L_{\mu}^{3}+21.3333 L_{\mu}^{4}\right. \\
& -\left(22884.9+10052.4 L_{\mu}-3024.01 L_{\mu}^{2}-444.744 L_{\mu}^{3}+85.3333 L_{\mu}^{4}\right) \xi(1-\xi) \\
& \left.+\left(50837.4+35185.8 L_{\mu}+4586.08 L_{\mu}^{2}-276.734 L_{\mu}^{3}-170.667 L_{\mu}^{4}\right)(\xi(1-\xi))^{2}\right] \beta^{4} \\
& +\mathcal{O}\left(\beta^{6}\right) .
\end{aligned}
$$

We observe that the dependence on $\beta$ and on $\xi$ in the formulas above is polynomial. Moreover, $\beta$ enters only via powers of $\beta^{2}$ and $\xi$ only via powers of $\xi(1-\xi)$. The latter dependence explicitely reflects the symmetry of $A$ under exchange of forward and backward directions, $\xi \rightarrow 1-\xi$. All of the logarithmic terms $\ln \beta, \ln \xi, \ln (1-\xi), \ln (1-2 \xi), \ldots$, which are indeed present in the expansion of individual HPLs, cancel out in the final expressions. Thus, the coefficient $A$ is finite at threshold. The expansion presented here could be used in the future for the calculation of logarithmically enhanced leading colour terms near the $t \bar{t}$ production threshold. 

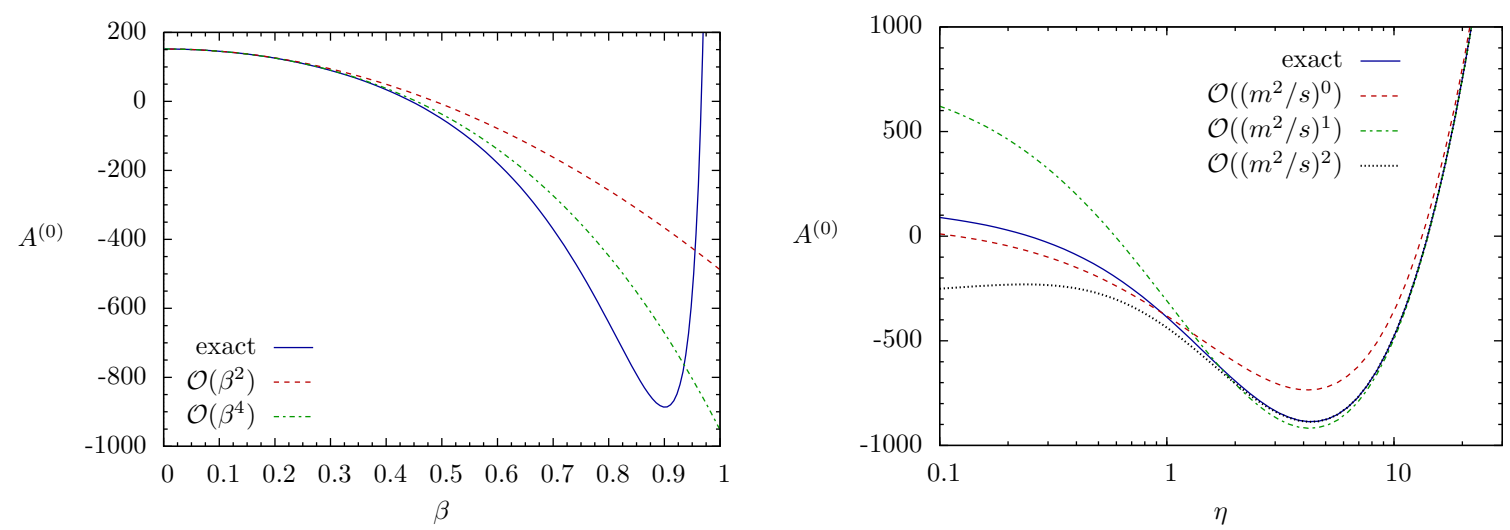

Figure 4. Left: finite part of the coefficient $A$ as a function of $\beta \equiv \sqrt{1-4 m^{2} / s}$ for $\xi=1 / 2$. Right: finite part of the coefficient $A$ as a function of the variable $\eta \equiv s /\left(4 m^{2}\right)-1$ for $\phi=1 / 2$. In both cases we used the normalization that factors out the term $\left[(4 \pi)^{\varepsilon} e^{-\gamma \varepsilon}\right]^{2}$ and set $\mu=m$.

In figure 4, we show the comparison between the exact expression of the coefficient $A$ and the expansions in the two regimes: threshold expansion on the left hand side and small-mass expansion on the right hand side. For the small-mass expansion we consider the limit $m^{2} / s \rightarrow 0$ for $\phi=-\left(t-m^{2}\right) / s=$ const and neglect terms suppressed by powers of $\mathrm{m}^{2} / \mathrm{s}$ larger than a given order. This prescription preserves the symmetry of the result under exchange of forward and backward directions, $\phi \rightarrow 1-\phi$, for a given order of the expansion.

With the arXiv submission of this paper, we provide algebraic expressions and numerical implementations of the threshold expansion up to order $\beta^{5}$ included and of the small mass expansion up to order $\left(\mathrm{m}^{2} / \mathrm{s}\right)^{2}$ included.

\section{Conclusions and outlook}

In this paper, we presented the analytic calculation of the two-loop leading color corrections to the heavy-quark production matrix element in the gluon fusion channel. The diagrams required to calculate this coefficient are all planar. The result presented here retains the exact heavy-quark mass dependence; no assumptions on the hierarchy between the mass scales involved in the problem was made. The formula for the coefficient $A$ in eq. (2.6), which was obtained in this work, was validated against analytic results valid in the smallmass region [71]. In addition, we found analytic agreement with the exact expression for the IR poles which was obtained in [93].

Our result represents a gauge invariant sub-set of the full two-loop corrections to the partonic process $g g \rightarrow t \bar{t}$. In order to complete the analytic calculation of the twoloop corrections, it is necessary to calculate all of the fermionic diagrams, and the nonplanar gluonic diagrams. A large part of the non-leading color coefficients in eq. (2.6) can be calculated within the same calculational framework employed here. However, it is known that some of the two-loop diagrams appearing in the gluon fusion channel cannot be expressed in terms the HPLs functional basis. For example, some of the diagrams 
with a closed heavy-quark loop involve a "sunrise"-type subtopology with three equal massive propagators and an external momentum which is not on the mass shell of the internal propagators. Such a three-propagator graph can be written only in terms of elliptic integrals [125]. The use of numerical [72] or seminumerical $[126,127]$ methods could thus be unavoidable in the evaluation of these diagrams.

In order to obtain NNLO predictions for the total $t \bar{t}$ production cross section and for differential distributions, it is necessary to combine the two-loop virtual corrections with the already available one-loop corrections to the $t \bar{t}+(1$ parton $)$ process and with the tree-level matrix elements for the process $t \bar{t}+(2$ partons $)$ [49-52]. These diagrams with additional partons in the final state contribute to infrared-divergent configurations where up to two partons can become unresolved. Their implementation requires the application of a NNLO subtraction method. The methods presently available [53-67] do not provide yet the full counterterms for a $2 \rightarrow 2$ hadronic process involving massive partons. Two subtraction methods for the NNLO calculation of the top-quark pair production cross section were outlined recently $[68,69]$.

In the light of these advancements, the calculation of the full NNLO corrections to top quark pair production in hadronic collisions is gradually becoming feasible.

\section{Acknowledgments}

Part of the algebraic manipulations of this work were done using FORM [128].

R. B. wishes to thank the Center for Theoretical Physics of New York City College of Technology for the kind hospitality during a part of this work. A. v. M. wishes to thank the Institute for Theoretical Physics of the University of Bielefeld for the kind hospitality during a part of this work and Y. Schröder for stimulating discussions.

The work of R. B. is supported by the Theory-LHC-France initiative of CNRS/IN2P3 and partly funded by the Center for Theoretical Physics of New York City College of Technology. T. G. and A. v. M. are supported by the Schweizer Nationalfonds (grants 200020-126691 and 200020-124773). The work of C. St. was supported by the Deutsche Forschungsgemeinschaft (DFG SCHR 993/2-1).

Open Access. This article is distributed under the terms of the Creative Commons Attribution Noncommercial License which permits any noncommercial use, distribution, and reproduction in any medium, provided the original author(s) and source are credited.

\section{References}

[1] CDF AND D0 collaboration, Combination of CDF and D0 results on the mass of the top quark, arXiv: 1007.3178 [SPIRES].

[2] CDF collaboration, T. Aaltonen et al., Top quark mass measurement in the lepton + jets channel using a matrix element method and in situ jet energy calibration, Phys. Rev. Lett. 105 (2010) 252001 [arXiv:1010.4582] [SPIRES]. 
[3] D0 collaboration, V.M. Abazov et al., Combination of t $\bar{t}$ cross section measurements and constraints on the mass of the top quark and its decays into charged Higgs bosons,

Phys. Rev. D 80 (2009) 071102 [arXiv:0903.5525] [SPIRES].

[4] CDF collaboration, T. Aaltonen et al., First measurement of the ratio $\sigma_{t \bar{t}} / \sigma_{Z / \gamma^{*} \rightarrow l l}$ and precise extraction of the $t \bar{t}$ cross section, Phys. Rev. Lett. 105 (2010) 012001 [arXiv: 1004.3224] [SPIRES].

[5] CDF collaboration, T. Aaltonen et al., First measurement of the $t \bar{t}$ differential cross section $d \sigma / d M_{t \bar{t}}$ in $p \bar{p}$ collisions at $\sqrt{s}=1.96$ TeV, Phys. Rev. Lett. 102 (2009) 222003 [arXiv:0903.2850] [SPIRES].

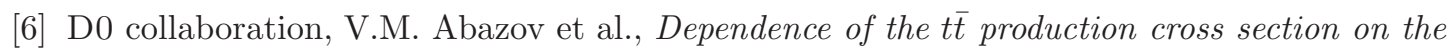
transverse momentum of the top quark, Phys. Lett. B 693 (2010) 515 [arXiv:1001.1900] [SPIRES].

[7] R. Frederix, Top quark phenomenology, arXiv:1009.6199 [SPIRES].

[8] W. Bernreuther, Keynote: some remarks on top, arXiv:1008.3819 [SPIRES].

[9] W. Bernreuther, Top quark physics at the LHC, J. Phys. G 35 (2008) 083001 [arXiv:0805.1333] [SPIRES].

[10] P. Nason, S. Dawson and R.K. Ellis, The total cross-section for the production of heavy quarks in hadronic collisions, Nucl. Phys. B 303 (1988) 607 [SPIRES].

[11] P. Nason, S. Dawson and R.K. Ellis, The one particle inclusive differential cross-section for heavy quark production in hadronic collisions, Nucl. Phys. B 327 (1989) 49 [Erratum ibid. B 335 (1990) 260] [SPIRES].

[12] W. Beenakker, H. Kuijf, W.L. van Neerven and J. Smith, QCD corrections to heavy quark

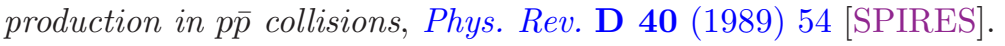

[13] W. Beenakker, W.L. van Neerven, R. Meng, G.A. Schuler and J. Smith, QCD corrections to heavy quark production in hadron hadron collisions, Nucl. Phys. B 351 (1991) 507 [SPIRES].

[14] M.L. Mangano, P. Nason and G. Ridolfi, Heavy quark correlations in hadron collisions at next-to-leading order, Nucl. Phys. B 373 (1992) 295 [SPIRES].

[15] J.G. Korner and Z. Merebashvili, One-loop corrections to four-point functions with two external massive fermions and two external massless partons, Phys. Rev. D 66 (2002) 054023 [hep-ph/0207054] [SPIRES].

[16] W. Bernreuther, A. Brandenburg, Z.-G. Si and P. Uwer, Top quark pair production and decay at hadron colliders, Nucl. Phys. B 690 (2004) 81 [hep-ph/0403035] [SPIRES].

[17] M. Czakon and A. Mitov, Inclusive heavy flavor hadroproduction in NLO QCD: the exact analytic result, Nucl. Phys. B 824 (2010) 111 [arXiv:0811.4119] [SPIRES].

[18] K. Melnikov and M. Schulze, NLO QCD corrections to top quark pair production and decay at hadron colliders, JHEP 08 (2009) 049 [arXiv:0907.3090] [SPIRES].

[19] W. Bernreuther and Z.-G. Si, Distributions and correlations for top quark pair production and decay at the Tevatron and LHC, Nucl. Phys. B 837 (2010) 90 [arXiv:1003.3926] [SPIRES].

[20] W. Beenakker et al., Electroweak one loop contributions to top pair production in hadron colliders, Nucl. Phys. B 411 (1994) 343 [SPIRES]. 
[21] J.H. Kühn, A. Scharf and P. Uwer, Electroweak corrections to top-quark pair production in quark-antiquark annihilation, Eur. Phys. J. C 45 (2006) 139 [hep-ph/0508092] [SPIRES].

[22] J.H. Kühn, A. Scharf and P. Uwer, Electroweak effects in top-quark pair production at hadron colliders, Eur. Phys. J. C 51 (2007) 37 [hep-ph/0610335] [SPIRES].

[23] W. Bernreuther, M. Fücker and Z.-G. Si, Weak interaction corrections to hadronic top quark pair production, Phys. Rev. D 74 (2006) 113005 [hep-ph/0610334] [SPIRES].

[24] W. Bernreuther, M. Fücker and Z.-G. Si, Weak interaction corrections to hadronic top quark pair production: contributions from quark-gluon and $b \bar{b}$ induced reactions, Phys. Rev. D 78 (2008) 017503 [arXiv:0804.1237] [SPIRES].

[25] E. Laenen, J. Smith and W.L. van Neerven, All order resummation of soft gluon contributions to heavy quark production in hadron hadron collisions,

Nucl. Phys. B 369 (1992) 543 [SPIRES].

[26] E. Laenen, J. Smith and W.L. van Neerven, Top quark production cross-section, Phys. Lett. B 321 (1994) 254 [hep-ph/9310233] [SPIRES].

[27] E.L. Berger and H. Contopanagos, Perturbative gluon resummation of the top quark production cross-section, Phys. Lett. B 361 (1995) 115 [hep-ph/9507363] [SPIRES].

[28] E.L. Berger and H. Contopanagos, The perturbative resummed series for top quark production in hadron reactions, Phys. Rev. D 54 (1996) 3085 [hep-ph/9603326] [SPIRES].

[29] E.L. Berger and H. Contopanagos, Threshold resummation of the total cross-section for heavy quark production in hadronic collisions, Phys. Rev. D 57 (1998) 253 [hep-ph/9706206] [SPIRES].

[30] S. Catani, M.L. Mangano, P. Nason and L. Trentadue, The top cross-section in hadronic collisions, Phys. Lett. B 378 (1996) 329 [hep-ph/9602208] [SPIRES].

[31] S. Catani, M.L. Mangano, P. Nason and L. Trentadue, The resummation of soft gluon in hadronic collisions, Nucl. Phys. B 478 (1996) 273 [hep-ph/9604351] [SPIRES].

[32] N. Kidonakis and G.F. Sterman, Subleading logarithms in QCD hard scattering, Phys. Lett. B 387 (1996) 867 [SPIRES].

[33] N. Kidonakis and G.F. Sterman, Resummation for QCD hard scattering, Nucl. Phys. B 505 (1997) 321 [hep-ph/9705234] [SPIRES].

[34] N. Kidonakis, G. Oderda and G.F. Sterman, Evolution of color exchange in QCD hard scattering, Nucl. Phys. B 531 (1998) 365 [hep-ph/9803241] [SPIRES].

[35] E. Laenen, G. Oderda and G.F. Sterman, Resummation of threshold corrections for single particle inclusive cross-sections, Phys. Lett. B 438 (1998) 173 [hep-ph/9806467] [SPIRES].

[36] R. Bonciani, S. Catani, M.L. Mangano and P. Nason, NLL resummation of the heavy-quark hadroproduction cross-section, Nucl. Phys. B 529 (1998) 424 [Erratum ibid. B 803 (2008) 234] [hep-ph/9801375] [SPIRES].

[37] R. Bonciani, S. Catani, M.L. Mangano and P. Nason, Sudakov resummation of multiparton QCD cross sections, Phys. Lett. B 575 (2003) 268 [hep-ph/0307035] [SPIRES].

[38] M. Czakon and A. Mitov, On the soft-gluon resummation in top quark pair production at hadron colliders, Phys. Lett. B 680 (2009) 154 [arXiv:0812.0353] [SPIRES].

[39] M. Beneke, P. Falgari and C. Schwinn, Soft radiation in heavy-particle pair production: all-order colour structure and two-loop anomalous dimension, Nucl. Phys. B 828 (2010) 69 [arXiv: 0907.1443] [SPIRES]. 
[40] M. Czakon, A. Mitov and G.F. Sterman, Threshold resummation for top-pair hadroproduction to next-to-next-to-leading log, Phys. Rev. D 80 (2009) 074017 [arXiv: 0907.1790] [SPIRES].

[41] V. Ahrens, A. Ferroglia, M. Neubert, B.D. Pecjak and L.L. Yang, Top-quark pair production beyond next-to-leading order, Nucl. Phys. Proc. Suppl. 205-206 (2010) 48 [arXiv:1006.4682] [SPIRES].

[42] A. Ferroglia, M. Neubert, B.D. Pecjak and L.L. Yang, Infrared singularities and soft gluon resummation with massive partons, Nucl. Phys. Proc. Suppl. 205-206 (2010) 98 [arXiv: 1006 .4680] [SPIRES].

[43] N. Kidonakis, Next-to-next-to-leading soft-gluon corrections for the top quark cross section and transverse momentum distribution, Phys. Rev. D 82 (2010) 114030 [arXiv:1009.4935] [SPIRES].

[44] S. Moch and P. Uwer, Theoretical status and prospects for top-quark pair production at hadron colliders, Phys. Rev. D 78 (2008) 034003 [arXiv:0804.1476] [SPIRES].

[45] U. Langenfeld, S. Moch and P. Uwer, Measuring the running top-quark mass, Phys. Rev. D 80 (2009) 054009 [arXiv:0906.5273] [SPIRES].

[46] U. Langenfeld, S. Moch and P. Uwer, New results for $t \bar{t}$ production at hadron colliders, arXiv:0907.2527 [SPIRES].

[47] M. Cacciari, S. Frixione, M.L. Mangano, P. Nason and G. Ridolfi, Updated predictions for the total production cross sections of top and of heavier quark pairs at the Tevatron and at the LHC, JHEP 09 (2008) 127 [arXiv:0804.2800] [SPIRES].

[48] N. Kidonakis and R. Vogt, The theoretical top quark cross section at the Tevatron and the LHC, Phys. Rev. D 78 (2008) 074005 [arXiv:0805. 3844] [SPIRES].

[49] S. Dittmaier, P. Uwer and S. Weinzierl, NLO QCD corrections to t $\bar{t}+$ jet production at hadron colliders, Phys. Rev. Lett. 98 (2007) 262002 [hep-ph/0703120] [SPIRES].

[50] S. Dittmaier, P. Uwer and S. Weinzierl, Hadronic top-quark pair production in association with a hard jet at next-to-leading order QCD: phenomenological studies for the Tevatron and the LHC, Eur. Phys. J. C 59 (2009) 625 [arXiv:0810.0452] [SPIRES].

[51] G. Bevilacqua, M. Czakon, C.G. Papadopoulos and M. Worek, Dominant QCD backgrounds in Higgs boson analyses at the LHC: a study of $p p \rightarrow t \bar{t}+2$ jets at next-to-leading order, Phys. Rev. Lett. 104 (2010) 162002 [arXiv:1002.4009] [SPIRES].

[52] K. Melnikov and M. Schulze, NLO QCD corrections to top quark pair production in association with one hard jet at hadron colliders, Nucl. Phys. B 840 (2010) 129 [arXiv: 1004.3284] [SPIRES].

[53] T. Binoth and G. Heinrich, An automatized algorithm to compute infrared divergent multi-loop integrals, Nucl. Phys. B 585 (2000) 741 [hep-ph/0004013] [SPIRES].

[54] T. Binoth and G. Heinrich, Numerical evaluation of phase space integrals by sector decomposition, Nucl. Phys. B 693 (2004) 134 [hep-ph/0402265] [SPIRES].

[55] G. Heinrich, A numerical method for NNLO calculations, Nucl. Phys. Proc. Suppl. 116 (2003) 368 [hep-ph/0211144] [SPIRES].

[56] G. Heinrich, A numerical approach to infrared divergent multi-parton phase space integrals, Nucl. Phys. Proc. Suppl. 135 (2004) 290 [hep-ph/0406332] [SPIRES]. 
[57] G. Heinrich, Towards $e^{+} e^{-} \rightarrow 3$ jets at NNLO by sector decomposition, Eur. Phys. J. C 48 (2006) 25 [hep-ph/0601062] [SPIRES].

[58] G. Heinrich, Sector decomposition, Int. J. Mod. Phys. A 23 (2008) 1457 [arXiv:0803.4177] [SPIRES].

[59] A. Gehrmann-De Ridder, T. Gehrmann and G. Heinrich, Four-particle phase space integrals in massless QCD, Nucl. Phys. B 682 (2004) 265 [hep-ph/0311276] [SPIRES].

[60] C. Anastasiou, K. Melnikov and F. Petriello, A new method for real radiation at NNLO, Phys. Rev. D 69 (2004) 076010 [hep-ph/0311311] [SPIRES].

[61] D.A. Kosower, Multiple singular emission in gauge theories, Phys. Rev. D 67 (2003) 116003 [hep-ph/0212097] [SPIRES].

[62] A. Daleo, T. Gehrmann and D. Maître, Antenna subtraction with hadronic initial states, JHEP 04 (2007) 016 [hep-ph/0612257] [SPIRES].

[63] A. Gehrmann-De Ridder and M. Ritzmann, NLO antenna subtraction with massive fermions, JHEP 07 (2009) 041 [arXiv: 0904.3297] [SPIRES].

[64] A. Gehrmann-De Ridder, T. Gehrmann and E.W.N. Glover, Antenna subtraction at NNLO, JHEP 09 (2005) 056 [hep-ph/0505111] [SPIRES].

[65] E.W. Nigel Glover and J. Pires, Antenna subtraction for gluon scattering at NNLO, JHEP 06 (2010) 096 [arXiv: 1003.2824] [SPIRES].

[66] R. Boughezal, A. Gehrmann-De Ridder and M. Ritzmann, NNLO antenna subtraction with two hadronic initial states, PoS (RADCOR2009) 052 [arXiv:1001.2396] [SPIRES].

[67] S. Catani and M. Grazzini, An NNLO subtraction formalism in hadron collisions and its application to Higgs boson production at the LHC, Phys. Rev. Lett. 98 (2007) 222002 [hep-ph/0703012] [SPIRES].

[68] M. Czakon, A novel subtraction scheme for double-real radiation at NNLO, Phys. Lett. B 693 (2010) 259 [arXiv: 1005.0274] [SPIRES].

[69] C. Anastasiou, F. Herzog and A. Lazopoulos, On the factorization of overlapping singularities at NNLO, arXiv:1011.4867 [SPIRES].

[70] M. Czakon, A. Mitov and S. Moch, Heavy-quark production in massless quark scattering at two loops in QCD, Phys. Lett. B 651 (2007) 147 [arXiv:0705.1975] [SPIRES].

[71] M. Czakon, A. Mitov and S. Moch, Heavy-quark production in gluon fusion at two loops in QCD, Nucl. Phys. B 798 (2008) 210 [arXiv:0707.4139] [SPIRES].

[72] M. Czakon, Tops from light quarks: full mass dependence at two-loops in QCD, Phys. Lett. B 664 (2008) 307 [arXiv:0803.1400] [SPIRES].

[73] R. Bonciani, A. Ferroglia, T. Gehrmann, D. Maître and C. Studerus, Two-loop fermionic corrections to heavy-quark pair production: the quark-antiquark channel, JHEP 07 (2008) 129 [arXiv:0806.2301] [SPIRES].

[74] R. Bonciani, A. Ferroglia, T. Gehrmann and C. Studerus, Two-loop planar corrections to heavy-quark pair production in the quark-antiquark channel, JHEP 08 (2009) 067 [arXiv:0906.3671] [SPIRES].

[75] S. Laporta and E. Remiddi, The analytical value of the electron (g-2) at order alpha 3 in QED, Phys. Lett. B 379 (1996) 283 [hep-ph/9602417] [SPIRES]. 
[76] S. Laporta, High-precision calculation of multi-loop Feynman integrals by difference equations, Int. J. Mod. Phys. A 15 (2000) 5087 [hep-ph/0102033] [SPIRES].

[77] F.V. Tkachov, A theorem on analytical calculability of four loop renormalization group functions, Phys. Lett. B 100 (1981) 65 [SPIRES].

[78] K.G. Chetyrkin and F.V. Tkachov, Integration by parts: the algorithm to calculate $\beta$-functions in 4 loops, Nucl. Phys. B 192 (1981) 159 [SPIRES].

[79] A.V. Kotikov, Differential equations method: new technique for massive Feynman diagrams calculation, Phys. Lett. B 254 (1991) 158 [SPIRES].

[80] A.V. Kotikov, Differential equations method: the calculation of vertex type Feynman diagrams, Phys. Lett. B 259 (1991) 314 [SPIRES].

[81] A.V. Kotikov, Differential equation method: the calculation of $N$ point Feynman diagrams, Phys. Lett. B 267 (1991) 123 [SPIRES].

[82] E. Remiddi, Differential equations for Feynman graph amplitudes, Nuovo Cim. A 110 (1997) 1435 [hep-th/9711188] [SPIRES].

[83] M. Caffo, H. Czyz, S. Laporta and E. Remiddi, Master equations for master amplitudes, Acta Phys. Polon. B 29 (1998) 2627 [hep-th/9807119] [SPIRES].

[84] M. Caffo, H. Czyz, S. Laporta and E. Remiddi, The master differential equations for the 2-loop sunrise selfmass amplitudes, Nuovo Cim. A 111 (1998) 365 [hep-th/9805118] [SPIRES].

[85] T. Gehrmann and E. Remiddi, Differential equations for two-loop four-point functions, Nucl. Phys. B 580 (2000) 485 [hep-ph/9912329] [SPIRES].

[86] M. Argeri and P. Mastrolia, Feynman diagrams and differential equations, Int. J. Mod. Phys. A 22 (2007) 4375 [arXiv:0707.4037] [SPIRES].

[87] J.G. Körner, Z. Merebashvili and M. Rogal, One-loop amplitudes for four-point functions with two external massive quarks and two external massless partons up to $\mathcal{O}\left(\varepsilon^{2}\right)$, Phys. Rev. D 73 (2006) 034030 [hep-ph/0511264] [SPIRES].

[88] J.G. Körner, Z. Merebashvili and M. Rogal, $N N L O \mathcal{O}\left(\alpha_{s}^{4}\right)$ results for heavy quark pair production in quark-antiquark collisions: the one-loop squared contributions, Phys. Rev. D 77 (2008) 094011 [arXiv:0802.0106] [SPIRES].

[89] C. Anastasiou and S.M. Aybat, The one-loop gluon amplitude for heavy-quark production at NNLO, Phys. Rev. D 78 (2008) 114006 [arXiv:0809.1355] [SPIRES].

[90] B. Kniehl, Z. Merebashvili, J.G. Körner and M. Rogal, Heavy quark pair production in gluon fusion at next-to-next-to-leading $\mathcal{O}\left(\alpha_{s}^{4}\right)$ order: one-loop squared contributions, Phys. Rev. D 78 (2008) 094013 [arXiv:0809.3980] [SPIRES].

[91] T. Becher and M. Neubert, Infrared singularities of QCD amplitudes with massive partons, Phys. Rev. D 79 (2009) 125004 [Erratum ibid. D 80 (2009) 109901] [arXiv:0904.1021] [SPIRES].

[92] A. Ferroglia, M. Neubert, B.D. Pecjak and L.L. Yang, Two-loop divergences of scattering amplitudes with massive partons, Phys. Rev. Lett. 103 (2009) 201601 [arXiv:0907.4791] [SPIRES].

[93] A. Ferroglia, M. Neubert, B.D. Pecjak and L.L. Yang, Two-loop divergences of massive scattering amplitudes in non-abelian gauge theories, JHEP 11 (2009) 062 [arXiv:0908.3676] [SPIRES]. 
[94] P. Nogueira, Automatic Feynman graph generation, J. Comput. Phys. 105 (1993) 279 [SPIRES].

[95] A. von Manteuffel and C. Studerus, Reduze 2, to appear.

[96] C. Anastasiou and A. Lazopoulos, Automatic integral reduction for higher order perturbative calculations, JHEP 07 (2004) 046 [hep-ph/0404258] [SPIRES].

[97] A.V. Smirnov, Algorithm FIRE - Feynman Integral REduction, JHEP 10 (2008) 107 [arXiv:0807.3243] [SPIRES].

[98] C. Studerus, Reduze - Feynman Integral REduction in $\mathrm{C}++$, Comput. Phys. Commun. 181 (2010) 1293 [arXiv:0912.2546] [SPIRES].

[99] C.W. Bauer, A. Frink and R. Kreckel, Introduction to the GiNaC framework for symbolic computation within the C++ programming language, J. Symb. Comput. 33 (2002) 1, [cs/0004015] [SPIRES].

[100] M. Argeri, P. Mastrolia and E. Remiddi, The analytic value of the sunrise self-mass with two equal masses and the external invariant equal to the third squared mass, Nucl. Phys. B 631 (2002) 388 [hep-ph/0202123] [SPIRES].

[101] R. Bonciani, P. Mastrolia and E. Remiddi, Vertex diagrams for the QED form factors at the 2-loop level, Nucl. Phys. B 661 (2003) 289 [Erratum ibid. B 702 (2004) 359] [hep-ph/0301170] [SPIRES].

[102] R. Bonciani, P. Mastrolia and E. Remiddi, Master integrals for the 2-loop QCD virtual corrections to the forward-backward asymmetry, Nucl. Phys. B 690 (2004) 138 [hep-ph/0311145] [SPIRES].

[103] R. Bonciani, P. Mastrolia and E. Remiddi, QED vertex form factors at two loops, Nucl. Phys. B 676 (2004) 399 [hep-ph/0307295] [SPIRES].

[104] J. Fleischer, A.V. Kotikov and O.L. Veretin, Analytic two-loop results for selfenergy- and vertex-type diagrams with one non-zero mass, Nucl. Phys. B 547 (1999) 343 [hep-ph/9808242] [SPIRES].

[105] U. Aglietti and R. Bonciani, Master integrals with one massive propagator for the two-loop electroweak form factor, Nucl. Phys. B 668 (2003) 3 [hep-ph/0304028] [SPIRES].

[106] A.I. Davydychev and M.Y. Kalmykov, Massive Feynman diagrams and inverse binomial sums, Nucl. Phys. B 699 (2004) 3 [hep-th/0303162] [SPIRES].

[107] U. Aglietti and R. Bonciani, Master integrals with 2 and 3 massive propagators for the 2-loop electroweak form factor: planar case, Nucl. Phys. B 698 (2004) 277 [hep-ph/0401193] [SPIRES].

[108] R. Bonciani, G. Degrassi and A. Vicini, On the generalized harmonic polylogarithms of one complex variable, arXiv:1007.1891 [SPIRES].

[109] M. Czakon, J. Gluza and T. Riemann, Master integrals for massive two-loop Bhabha scattering in QED, Phys. Rev. D 71 (2005) 073009 [hep-ph/0412164] [SPIRES].

[110] G. Bell, Higher order QCD corrections in exclusive charmless B decays, arXiv:0705.3133 [SPIRES].

[111] R. Bonciani and A. Ferroglia, Two-loop QCD corrections to the heavy-to-light quark decay, JHEP 11 (2008) 065 [arXiv:0809.4687] [SPIRES]. 
[112] H.M. Asatrian, C. Greub and B.D. Pecjak, NNLO corrections to $\bar{B} \rightarrow X_{u} l \bar{\nu}$ in the shape-function region, Phys. Rev. D 78 (2008) 114028 [arXiv:0810.0987] [SPIRES].

[113] M. Beneke, T. Huber and X.Q. Li, Two-loop QCD correction to differential semi-leptonic $b \rightarrow u$ decays in the shape-function region, Nucl. Phys. B 811 (2009) 77 [arXiv:0810.1230] [SPIRES].

[114] A.B. Goncharov, Multiple polylogarithms, ciclotomy and modular complexes, Math. Res. Lett. 5 (1998) 497.

[115] D.J. Broadhurst, Massive 3-loop Feynman diagrams reducible to $S C^{*}$ primitives of algebras of the sixth root of unity, Eur. Phys. J. C 8 (1999) 311 [hep-th/9803091] [SPIRES].

[116] E. Remiddi and J.A.M. Vermaseren, Harmonic polylogarithms, Int. J. Mod. Phys. A 15 (2000) 725 [hep-ph/9905237] [SPIRES].

[117] T. Gehrmann and E. Remiddi, Numerical evaluation of harmonic polylogarithms, Comput. Phys. Commun. 141 (2001) 296 [hep-ph/0107173] [SPIRES].

[118] D. Maître, HPL, a Mathematica implementation of the harmonic polylogarithms, Comput. Phys. Commun. 174 (2006) 222 [hep-ph/0507152] [SPIRES].

[119] D. Maître, Extension of HPL to complex arguments, hep-ph/0703052 [SPIRES].

[120] J. Vollinga and S. Weinzierl, Numerical evaluation of multiple polylogarithms, Comput. Phys. Commun. 167 (2005) 177 [hep-ph/0410259] [SPIRES].

[121] T. Gehrmann and E. Remiddi, Two-loop master integrals for $\gamma^{*} \rightarrow 3$ jets: the planar topologies, Nucl. Phys. B 601 (2001) 248 [hep-ph/0008287] [SPIRES].

[122] T. Gehrmann and E. Remiddi, Two-loop master integrals for $\gamma^{*} \rightarrow 3$ jets: the non-planar topologies, Nucl. Phys. B 601 (2001) 287 [hep-ph/0101124] [SPIRES].

[123] T. Gehrmann and E. Remiddi, Numerical evaluation of two-dimensional harmonic polylogarithms, Comput. Phys. Commun. 144 (2002) 200 [hep-ph/0111255] [SPIRES].

[124] K. Melnikov and T. van Ritbergen, The three-loop on-shell renormalization of $Q C D$ and QED, Nucl. Phys. B 591 (2000) 515 [hep-ph/0005131] [SPIRES].

[125] S. Laporta and E. Remiddi, Analytic treatment of the two loop equal mass sunrise graph, Nucl. Phys. B 704 (2005) 349 [hep-ph/0406160] [SPIRES].

[126] S. Pozzorini and E. Remiddi, Precise numerical evaluation of the two loop sunrise graph master integrals in the equal mass case, Comput. Phys. Commun. 175 (2006) 381 [hep-ph/0505041] [SPIRES].

[127] U. Aglietti, R. Bonciani, L. Grassi and E. Remiddi, The two loop crossed ladder vertex diagram with two massive exchanges, Nucl. Phys. B 789 (2008) 45 [arXiv:0705.2616] [SPIRES].

[128] J.A.M. Vermaseren, New features of FORM, math-ph/0010025 [SPIRES]. 